Aims. To compare Lithium prescribing practices in a Psychiatry of Old Age (POA) Service in the North-West of Ireland among adults aged 65 years and over with best practice guidelines.

Method. Review of the literature informed development of audit standards for Lithium prescribing. These included National Institute for Clinical Excellent (NICE) 2014 guidelines, The British National Formulary (2019) and Maudsley Prescribing Guidelines (2018). Data were collected retrospectively, using an audit-specific data collection tool, from clinical files of POA team caseload, aged 65 years or more and prescribed Lithium over the past one year.

Result. At the time of the audit in February 2020, 18 patients were prescribed lithium, $67 \%$ female, average age 74.6 years. Of those prescribed Lithium; $50 \%(n=9)$ had a depression diagnosis, $44 \%(\mathrm{n}=8)$ had bipolar affective disorder $(\mathrm{BPAD})$ and $6 \%(\mathrm{n}=$ 1) had schizoaffective disorder.

$78 \%(\mathrm{n}=14)$ of patients were on track to meet, or had already met, the NICE standard of 3-monthly serum lithium level. Lithium levels were checked on average 4.5 times in past one year, average lithium level was $0.61 \mathrm{mmol} / \mathrm{L}$ across the group and $39 \%(n=7)$ had lithium level within recommended therapeutic range $(0.6-0.8 \mathrm{mmol} / \mathrm{L})$.

$83 \%(n=15)$ of patients met the NICE standards of 3 monthly renal tests, thyroid function test was performed in $89 \%(\mathrm{n}=16)$ and at least one serum calcium level was documented in $63 \%$ $(\mathrm{n}=15)$. Taking into consideration most recent blood test results, $100 \%(n=18)$ had abnormal renal function, $78 \%(n=7)$ had abnormal thyroid function and $60 \%(n=9)$ had abnormal serum calcium.

Half $(n=9)$ were initiated on lithium by POA service and of these, $56 \%(n=5)$ had documented renal impairment prior to initiation. Of patients on long term lithium therapy at time of referral $(n=9)$, almost half $(n=4)$ had a documented history of lithium toxicity.

Conclusion. The results of this audit highlight room for improvement in lithium monitoring of older adults attending POA service. Furthermore, all patients prescribed lithium had impaired renal function, half had abnormal calcium and two fifths had abnormal thyroid function. This is an important finding given the associations between those admitted to hospital with COVID-19 and comorbid kidney disease and increased risk of inpatient death.

Our findings highlight the need for three monthly renal function monitoring in older adults prescribed lithium given the additive adverse effects of increasing age and lithium on the kidney. Close working with specialised renal services to provide timely advice on renal management for those with renal impairment prescribed lithium is important to minimise adverse patient outcomes.

\footnotetext{
Improving the patient involvement in research and development on acute psychiatric wards - an audit and quality improvement project

\author{
Ioana Varvari ${ }^{1 \star}$, Hany El - Sayeh ${ }^{2}$, Shona McIlrae ${ }^{3}$ \\ and Susan Bonner ${ }^{4}$ \\ ${ }^{1}$ Psychiatry Registrar, Tees, Esk and Wear Valleys NHS Foundation \\ Trust; ${ }^{2}$ Consultant Psychiatrist and Director of Medical Education, \\ Tees, Esk and Wear Valleys NHS Foundation Trust; ${ }^{3}$ Consultant \\ Psychiatrist and Clinical Director,Tees, Esk and Wear Valleys NHS \\ Foundation Trust and ${ }^{4}$ Research nurse, Tees Esk and Wear Valleys \\ NHS foundation Trust
} ${ }^{\star}$ Corresponding author.
}

doi: 10.1192/bjo.2021.324
Aims. The local audit aimed at measuring awareness of research and development policies and implementation of local and national standards. Our findings generated a quality improvement project with two main objectives: first, improving patient approach and recruitment in research and second, improving trainee satisfaction within our trust.

Method. A cohort of new inpatient admissions was identified over a period of 4 weeks, between October 2019 and November 2019, on the two psychiatric wards at the Briary Wing, Harrogate District Hospital. Based on local and national standards, we designed and developed a qualitative (questionnaire) and quantitative (audit tool) approach that was aimed at both staff and patients. Our steps included: assessing awareness and implementation of standards, a retrospective collection of data on the wards, and analysis of the data in Microsoft Excel.

Result. Only one ward implemented the local guidance from which we identified a sample of 14 consecutive new admissions that were currently present on the ward and were able to answer our questions. 13 of those patients were noted as 'approached' on our visual board from which only 3 patients remembered reading a leaflet about research options in the admission pack, however, they have not been verbally informed. There was no process in place to assure the re-approaching of initially unwell patients or to follow up on discharge for those interested. Documentation was available in only 9 of the cases and was nonspecific: 'admission pack done'.

Conclusion. The awareness and understanding of Research and Development policies are poor and they are difficult to apply in practice in a busy inpatient environment without a clear process in place. This results in patients missing the opportunity to learn and understand more about research or to participate in ongoing studies. Quality improvement work needs to be done to improve patient recruitment in research in inpatient settings. Simple flow charts and stepwise processes as exemplified by our action plan have the potential to improve service quality, as well as patient and trainee satisfaction.

\section{Reducing high dose antipsychotic therapy (HDAT) in a community mental health team (CMHT)}

Richard Walsh ${ }^{1 *}$, Sonn Patel ${ }^{2}$, Valentina Loddo ${ }^{2}$, Rebecca Fahy ${ }^{3}$ and Elizabeth Walsh ${ }^{2}$

${ }^{1}$ School of Medicine, University College Dublin; ${ }^{2}$ Galway University Hospital and ${ }^{3}$ Semmelweis University

${ }^{\star}$ Corresponding author.

doi: 10.1192/bjo.2021.325

Aims. The consensus statement (CR190) of The Royal College of Psychiatrists states that the benefit of prescribing HDAT does not outweigh the risk of the increased side effect burden. HDAT is defined as the "daily dose of a single antipsychotic exceeding the upper limit for that drug as stated in the Summary of Product Characteristic (SPC) or British National Formulary (BNF)," and as the cumulative daily dose of two or more antipsychotics (for combined prescription). The prevalence of HDAT has been shown to vary widely and protocols for monitoring poorly implemented. In 2018 we completed a baseline survey of the prevalence of HDAT within our CMHT. We assessed our prescribing practice as compared to seven best practice audit criteria, which were adopted. Our aim is to resurvey closing the audit loop to 1) establish the current prevalence of HDAT and 2) assess the impact the intervention on prescribing practice. 
Method. Multi-disciplinary case notes for all registered patients were studied. A database was created including sociodemographic details, chart diagnosis, and medication. The proportion of patients prescribed antipsychotic medication was identified. The dose of each medication was converted into a percentage of BNF maximum recommended dose for that drug. For combined antipsychotic prescription, the cumulative dose was obtained adding the single percentages together. Exceeding 100\% was regarded as HDAT. All HDAT patients were assessed against identified audit criteria as outlined by the Humber NHS Foundation Trust.

Result. Of a total of 246 patients, 177 (72\%) were prescribed antipsychotic medication. Of these, 14 (8\%) were in receipt of HDAT. This compared to $68 \%$ prescribed antipsychotics and $9 \%$ in receipt of HDAT in the baseline audit. The average cumulative dose for every category (oral medication, depot and both) was calculated with a range from $1 \%$ to $168 \%$ (mean $=70 \%)$ for oral antipsychotic (single/combined), $1 \%$ to $193 \%$ (mean $=50 \%$ ) for depots and $20 \%$ to $257 \%$ (mean $=95 \%$ ) for combination of oral and depot. This compares with ranges of $1.6 \%$ to $215 \%$ (mean $=44.3 \%$ ) for oral antipsychotic (single/combined), $0.04 \%$ to $100 \%$ (mean $=25.8 \%)$ for depots and $21 \%$ to $425 \%$ (mean $=119.6 \%$ ) for combination of oral and depot in the baseline audit. Similar to the baseline survey no patient met all seven audit criteria but there was better adherence overall with best practice guidance. Blood and ECG monitoring were the most consistent parameters measured.

Conclusion. Lower HDAT was achieved post intervention. Results, whilst positive, indicate the need for ongoing audit to maintain best standards.

A review of required monitoring and management of physical health parameters in patients being treated with clozapine

Louisa Ward*, Charlotte Marriott, Giles Glass, Mariam Negm and Hannah Porter

worcestershire health and care trust

${ }^{\star}$ Corresponding author.

doi: $10.1192 /$ bjo.2021.326

Aims. To review available standards for physical health monitoring in people taking clozapine To audit current practice against standards To identify changes in practice and facilitate a re-audit to assess impact of any changes

Method. Standard: CG178 Psychosis and Schizophrenia in Adults: Prevention and Management - NICE, February 2014

Target:100\%

Exceptions: None

Sample: The original audit included all 58 patients from the Worcester clozapine clinic, as per October 2018. The re-audit reviewed a random sample of all patients attending the clozapine clinics in Worcester, Kidderminster and Redditch, as part of Worcestershire Health and Care NHS Trust, as per October 2019. A total of 66 patients were selected.

Data Source: Carenotes and ICE

Result. Areas of good practice:

Monitoring of $\mathrm{HbAlc}$ and $\mathrm{FBC}$ remains good

There has been an improvement in monitoring alcohol use, substance misuse and side effects

Areas requiring improvement:

There continues to be limited recording of respiratory rate

There has been a decline in recording temperature, BMI and concomitant therapies

Potential reasoning for missing data includes:
Staff not knowing the monitoring requirements, which is more likely to be an issue when staff members running the clinics change frequently

Monitoring being completed but not documented

Patients' refusal of monitoring

Data being recorded in alternative locations including general practice, without communication between services

Patients moving between teams or having inpatient stays may disrupt monitoring regime

Conclusion. LIMITATIONS

This audit assumes all patients involved to be on a stable dose of clozapine with routine monitoring

Some patients may have been transferred between teams or inpatients during the period of data collection

There is no scope to record when patients refuse monitoring

We may not have access to all notes such as those from general practice for data collection

\section{RECOMMENDATIONS}

Induction programme for junior doctors to include education on clozapine monitoring

Training for staff involved in clozapine clinics to ensure better understanding of monitoring requirements

Procurement of ECG machines for each site and relevant training for nursing and medical staff

Collaboration with GPs for shared data

Re-audit in 1 year

Service evaluation of weight gain in patients prescribed antipsychotics within the early intervention service

Louisa Ward*, Charlotte Marriott and Godwin Tong

worcestershire health and care trust

${ }^{\star}$ Corresponding author.

doi: 10.1192/bjo.2021.327

Aims. To assess physical health in patients under the Early Intervention Service, whom are prescribed antipsychotics.

To consider whether further intervention needs to be provided or promoted to improve physical health in this group.

Method. Assessment of carenotes database for all 63 patients on EIS caseload prescribed antipsychotics.

Result. Out of 47 patients studied, 20 were non-smokers at baseline. $25 \%$ of them ended up becoming smokers by the end of the study time.

Out of 47 patients studied 28 were non-drinkers at baseline. $32 \%$ of them ended up engaging in alcohol by the end of the study time.

Out of 47 patients studied, 38 patients had data available to record weight changes per year. Out of the 38 patients, 27 of them had positive weight change; average weight change was $+6.38 \mathrm{~kg}$ per year. The highest weight gain was $38.4 \mathrm{~kg}$, the highest weight lost was 47.3 kg.Out of 47 patients studied, 35 patients had data available to record BMI changes. Out of the 35 patients, 27 of them had positive BMI increases, average BMI change was +2.68 . The highest BMI increase was 12.84. The highest BMI decrease was 8.24.

Out of 47 patients studied, 11 patients had data available to record random glucose level changes. Out of the 11 patients, 7 of them had increased glucose levels, average glucose change were $+0.5 \mathrm{mmol} / \mathrm{l}$. The highest increase in glucose was $3.9 \mathrm{mmol} / \mathrm{l}$ and the highest drop in glucose was $2.6 \mathrm{mmol} / 1$.

Out of 47 patients studied, 19 patients had data available to record $\mathrm{HbAlc}$ levels. Out of the 19 patients, 10 of them had increased HbAlc levels, with the average change being $+0.31 \mathrm{mmol} / \mathrm{mol}$. The highest increase in $\mathrm{HbAlc}$ levels was $5 \mathrm{mmol} / \mathrm{mol}$ and the highest drop in $\mathrm{HbAlc}$ levels was $3 \mathrm{mmol} / \mathrm{mol}$. 